\title{
Determination the Specific Parameters for Uniform Power Separation of Optical Signals
}

\author{
Al-Gawagzeh Mohammed Yousef
}

\begin{abstract}
This paper will study the importance of using the features of anisotropic medium to decrease the effect of Attenuation and dispersion on the transmitted signals in the fiber optic system due to the power exchange between waves, a calculation for specific cases were made. Also to determine the half-length of the beat region $\xi_{1}$ for a certain composition of the spiral single mode optical fibers depending on the parameters of the spiral to construct spiral single mode optical fibers based on photo elasticity. Also in this paper we will try to define specific parameters for uniform power separation of the optical signal.
\end{abstract}

Key words: Optical fiber, spiral, photo elasticity, power separation.

\section{I.INTRODUCTION}

The single modes optical fibers were strongly used in many fields of science and techniques. The debugged technology of such fibers assumes their application exclusively in optical systems were there parameters strictly correspond to characteristics of fiber, in practical situations an actual optical fiber is not absolutely perfect, and it is neither completely axially-symmetric nor perfectly straight. In addition, the fiber material is often assumed to be nominally isotropic, in which the refractive index is the same regardless of the direction of the polarization of the electric field.

The implementation of anisotropic components in the construction of optical fiber transmission systems represents a scientific interest. But there are some difficulties in transmission process such uniform separation of signal power and minimization the losses in directional coupler, however using the features of anisotropic media will improve these characteristics. Several methods used to create an anisotropic optical fiber, one of these methods is photo elasticity by the deposition of the fiber in a spiral form .The double refraction in an anisotropic medium, owing to ordinary and an extraordinary wave that deviates at different angles with relative to the fiber axis can be used for separation the signal power.

The author is with the Balqa Applied University, Jordan, Faculty of Engineering, Power Engineering Department,

gogazeh@bau.edu.jo
To implement and design the directional couplers, and due to photo elasticity, It is necessary to study many features such behavior of ordinary $\left(H E_{11}^{o}\right)$ and extraordinary ( $H E_{11}^{e}$ ) waves in an anisotropic medium, the elements of dielectric permittivity tensor, the characteristics of power exchange between the main waves ( $H E_{11}^{e}$ and $H E_{11}^{o}$ ), and the effect of photo elasticity on the polarized dispersion. Because the single-mode fibers widely used in present optical communication systems, the polarization states of the input and the output light beams do not match, since the polarization of the output light beam is unstable. By contrast, polarized mode (PM) fibers maintain the state of polarization of a light beam passing through them. PM fibers are imperative for obtaining a stable output in interferometric fiber optical sensors. In optical communication devices the use of PM fiber becomes mandatory when performing any polarized waves operations, e.g., for polarization combining. There are many applications where the polarization of the light is required to be stable and well defined, such as coupling to the integrated optical circuits, interferometric sensors, and coherent optical communication systems.

One basic mode (HE11) (for simplicity we neglect the double refraction fiber) the situation becomes more complicated for double-refraction fibers, and at the same time in the real case on the fiber input, the energy of initial bunch is non-uniformly distributed on their own modes and the weights of modes are rigidly connected with conditions of optical fiber system excitation. And here again we collide with the problem of weighing of own modes.

The features of spiral single mode optical fibers (SSMOF) and the possibility of implementing this fiber in the system of transfer, where the principle of operation is based on anisotropy using for uniform power separation have not been deeply investigated.so its necessary to change the specific parameters of above features taking in consideration the anisotropy influence. 


\section{Related works}

There are many scientific researches devoted to this issue. For example in $[2,7]$ during the power exchange between the main waves (ordinary and extraordinary), the transient attenuation was studied and the range of losses in the parameter $A=\rho / 4 \pi R^{(\rho-\text { spiral step, } R \text { - spiral radius })}$ was determined. In [8] it was analyzed the implementation of angular dispersion property in the anisotropy medium for the purpose of signal power separation with consideration the double refraction.

In $[5,7]$ the parameters of an anisotropic self-focusing optical fiber were calculated. In $[1,11]$ the polarized dispersion of optical fiber that subjected to the influence of mechanical stresses have been carried out, and expressions have been obtained for its calculation. In $[2,3,4,6,9$, 12] the polarized modal dispersion in the wavelength range (1510 to $1645 \mathrm{~nm}$ ) due to the bent fiber has been measured.

However, in the above mentioned researches, the condition of coupling power between the main waves in the directional coupler due to photo elasticity of optical fiber was not defined.

The purpose of this paper is to determine the specific parameters for designing the directional couplers for uniform power separation of optical signal.

\section{II.MATERIALS AND METHODS}

According to the Mauls law, the intensity of ordinary $I_{o}$ and extraordinary $I_{e}$ waves is related to the angles $\left(\beta_{o}, \beta_{e}\right.$ ) of optical axis and the tension vectors $\vec{E}_{e}, \vec{E}_{o}$ as following [8]:

$$
I_{o}=I \cdot \sin ^{2} \cdot \beta_{o} ; I_{e}=I \cdot \cos ^{2} \cdot \beta_{e}
$$

Where $I$ - the radiation intensity before the fiber bending.

Obviously, with a uniform power distribution $\left(I_{o}=I_{e}\right)$, it is necessary to consider that $\left(\beta_{o}=\beta_{e}=45^{0}\right)$ as shown on the next figure.

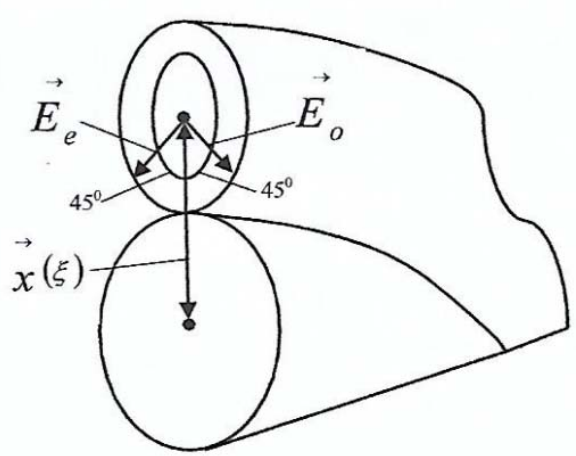

Figure 1 - polarization of main waves when a signal entering into the (SSMOF) for equal power separation

Where $\vec{E}_{o}, \vec{E}_{e}$ - the intensity vectors of ordinary and extraordinary waves.

$\vec{X}(\xi)$ - Optical axis.

The power ratio between the fundamental waves $\left(H E_{11}^{e}\right.$ and $H E_{11}^{o}$ ) must be equal to unity. When a wave is entered into an anisotropic medium, the birefringence occurs and at the initial time moment under the condition ( $\beta_{o}=\beta_{e}=45^{0}$ ), the power of extraordinary wave is equal to the power of ordinary.when the signal passing through the (SSMOF), the power is exchanged between main waves and the power transfer direction changes to the opposite after passing the length of (SSMOF) which equal to the half- beat length $\left(\xi_{1}\right)$.

Depending on the spiral parameters, the pumped power value from $H E_{11}^{e}$ wave to the $H E_{11}^{o}$ wave could be differing from the power that is pumped from the $H E_{11}^{o}$ wave to the $H E_{11}^{e}$ wave (inequality). The importance of this task is to achieve an equality between transient attenuation $\left(\alpha_{n 1}^{o e}\left(\xi_{1}\right)\right.$ and $\left.\alpha_{n 1}^{e o}\left(\xi_{1}\right)\right)$, which mean that the transmitted optical power by one of the main waves after passing of the half- beat length of the $\left(\xi_{1}\right)$ should be equal to the received optical power after passing the next section of $\xi_{1}$. In this case, after passing the length of $\left(2 \xi_{1}\right)$ the power of extraordinary wave $H E_{11}^{e}$ is equal to the power of the ordinary wave $H E_{11}^{o}$.

Using the function of photo elasticity of power transfer from extraordinary wave to the ordinary:

$$
f(A)^{e o}=\frac{A \sqrt{1+A^{2}}}{\left|A^{2}+A-1\right|} \cdot\{.\}^{e o}
$$


And the function of photo elasticity when the power transferring from an ordinary wave to an extraordinary:

$$
f(A)^{o e}=\frac{A \sqrt{1+A^{2}}}{\left|A^{2}+A-1\right|} \cdot\{\cdot\}^{o e}
$$

Where $\{.\}^{e o}$ - the expression in formula (29), and $\{.\}^{o e}$ - the expression in the formula (35) in [7].

These functions show the dependence of bending geometry of the optical fiber (fractional expressions) and the polarization angles of (SSMOF) (expressions in curly brackets).
Using the results that investigated and carried out in $[2,7]$, we obtain the condition of uniform power distribution between the main waves ( $H E_{11}^{e}$ and $H E_{11}^{o}$ ):

$\alpha_{n 1}^{e o}\left(\xi_{1}\right)=\alpha_{n 1}^{o e}\left(\xi_{1}\right)$

If we apply expressions (2), (3), and also the calculated formulas for the transition attenuation that obtained in $[2$, 7], then the expression (4) can be reduces to the form:

$$
f(A)^{e o}=f(A)^{o e}
$$

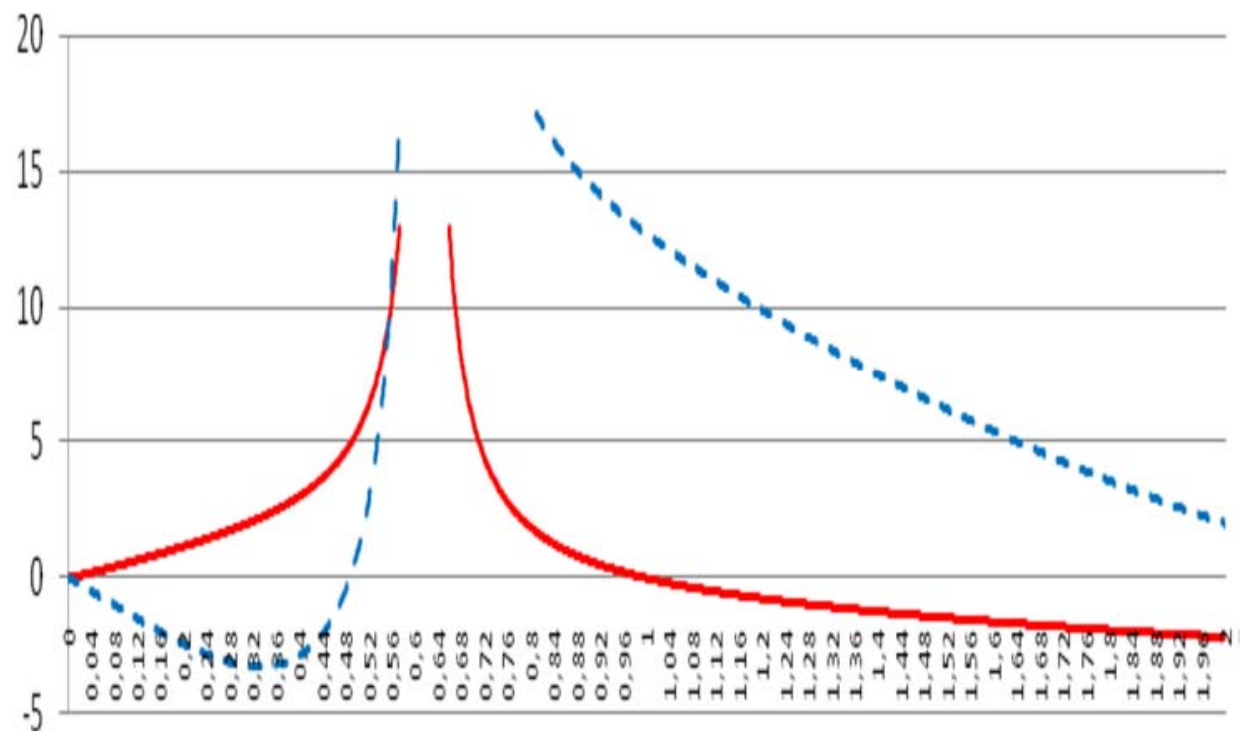

Figure 2 - Dependences of the photo elasticity functions $f(A)^{e o}$

(Solid line) and $f(A)^{o e}$ (dashed line) on the parameter $(A)$

Figure. 2 show the dependences of the functions $f(A)^{e o}$ and $f(A)^{o e}$ on the dimensionless parameter $(A)$.

The curves of $f(A)^{e o}$ and $f(A)^{o e}$ gives a possibility to determine for which values of $(A)$ parameter the transient attenuation occurs, so we can see that for a positive value of $f(A)^{e o}$, the power of the $H E_{11}^{e}$ waves will be pumped to the wave $H E_{11}^{o}$, and for $f(A)^{e o} \leq 0$ practically there is no power transfer, similarly for $f(A)^{o e}$.

As seen from the graph of Fig. 2, the condition for expression (5) is achieved for the values of A (from 0 to 2): $\mathrm{A}=0$ (bent along the arc of the circumference of optical fiber), $\mathrm{A}=0.553, \mathrm{~A}>2$.
The half- beat length region is calculated in [7]:

$$
\xi=\frac{\sqrt{2} \lambda \cdot n_{1} \cdot R}{w} \cdot \frac{\left(1+4 A^{2}\right) \cdot \sqrt{1+A^{2}}}{\left|A^{2}+A-1\right|}
$$

For determining the beat half-length region for a certain composition of the (SSMOF) with value of parameter (A) that corresponds to a uniform distribution of the signal power, it is necessary to determine the following (SSMOF) parameters: spiral radius, twisting step, the turns number, the length of the optical fiber, the length of the spiral where the optical fiber is deposited, and also the deviation angle $(\alpha)$ between $H E_{11}^{e}$ and $H E_{11}^{o}$ waves. 
Since the spatial spreading of the waves must be carried out, then the length of the optical fiber and the length of the spiral are:

$$
l=2 . \xi_{1} \cdot m ; \quad s=k . \rho
$$

where $l$-is the optical fiber length, $m$ - is an integer, $s$-is the spiral length where the optical fiber is deposited, $k$-is the number of spiral turns.

Considering the length of optical fiber that spent on one turn in the form of a hypotenuse a rectangular, and the length of the cylinder cross section where the winding is performed, the total length of the optical fiber is:

$$
l=k \sqrt{\rho^{2}+(2 \pi R)^{2}}
$$

\section{III.RESULTS AND DESCUSSION}

Using the expression (7), the turn's number of (SSMOF) can be defined as:

$$
k=\frac{2 \xi_{1} \cdot m}{\sqrt{\rho^{2}+(2 \pi R)^{2}}}
$$

Figure (3) shows the construction of (SSMOF) that necessary for uniform signal power distribution.

Table.1 show the parameters calculations results with a uniform power separation for some compositions of optical fiber with a core radius of $4.5 \mu \mathrm{m}, \mathrm{m}=1, A=0.553$ and a wavelength of $1.565 \mu \mathrm{m}$

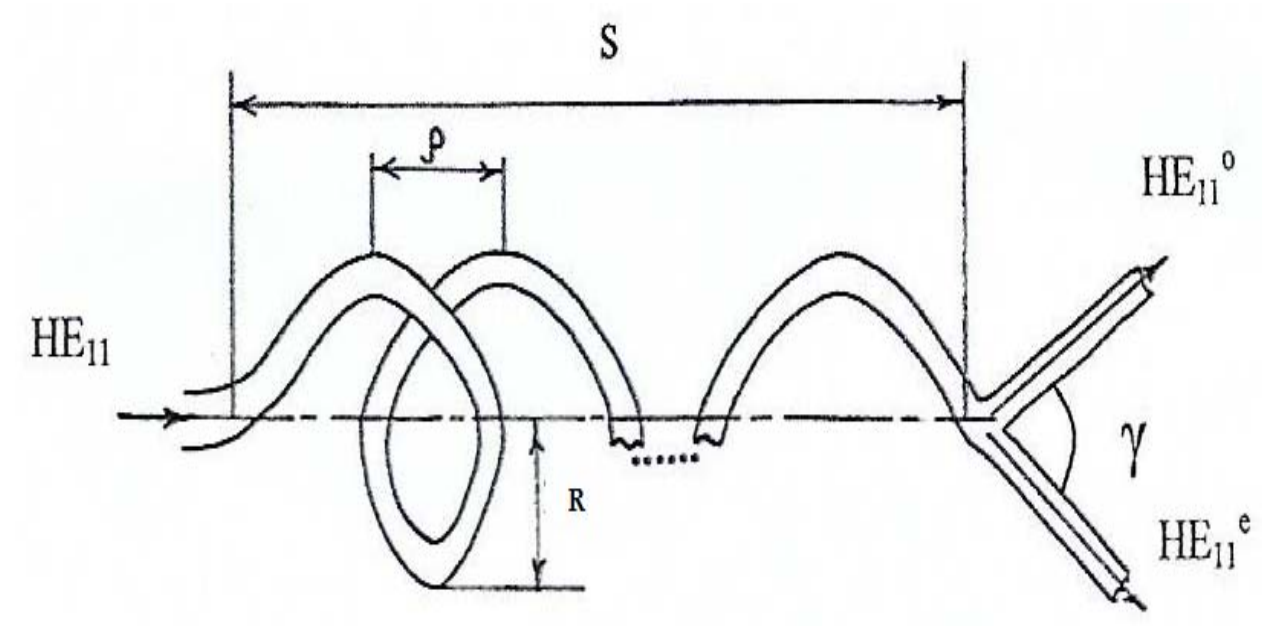

Figure 3 - Power distribution based on photo elasticity 
Table 1.Parameters of optical fibers that deposited in a spiral line for equal separation of signal power

\begin{tabular}{|c|c|c|c|c|c|}
\hline Optical fiber content & $\begin{array}{l}\text { number of } \\
\text { turns of the } \\
\text { helix }(k)\end{array}$ & $\begin{array}{l}\text { Beat length } \\
\left(2 \xi_{1}\right), \mathrm{m}\end{array}$ & $\begin{array}{c}\text { Parameter } \\
A\end{array}$ & $\begin{array}{c}\text { Pitch twist } \\
\rho, \mathrm{m}\end{array}$ & $\begin{array}{c}\text { Radius of } \\
\text { modal field } \\
\quad H E_{11} \\
W, \mu \mathrm{m}\end{array}$ \\
\hline $\begin{array}{l}\text { 1.core } 100 \% \mathrm{SiO}_{2} \\
\text { shell } 1 \% \mathrm{~F}, 99 \% \mathrm{SiO}_{2}\end{array}$ & 2.834152 & 0.132692 & 0.553 & 0.034728 & 4.333 \\
\hline $\begin{array}{l}\text { 2.core } 2.2 \% \mathrm{GeO}_{2}, 3.3 \% \mathrm{~B}_{2} \mathrm{O}_{3} \text {, } \\
94.5 \% \mathrm{SiO}_{2} \\
\text { shell } 1 \% \mathrm{~F}, 99 \% \mathrm{SiO}_{2}\end{array}$ & 3.375232 & 0.158025 & 0.553 & 0.034728 & 3.644 \\
\hline $\begin{array}{l}\text { 3.core } 9.1 \% \mathrm{P}_{2} \mathrm{O}_{5}, 90.9 \% \mathrm{SiO}_{2} \\
\text { shell } 3.5 \% \mathrm{~B}_{2} \mathrm{O}_{3}, 96.5 \% \mathrm{SiO}_{2}\end{array}$ & 4.810408 & 0.225218 & 0.553 & 0.034728 & 2.579 \\
\hline $\begin{array}{l}\text { 4.core } 16.9 \% \mathrm{Na}_{2} \mathrm{O}, 32.5 \% \\
\mathrm{~B}_{2} \mathrm{O}_{3}, 50.6 \% \mathrm{SiO}_{2} \\
\text { shell } 1 \% \mathrm{~F}, 99 \% \mathrm{SiO}_{2}\end{array}$ & 7.591813 & 0.355441 & 0.553 & 0.034728 & 1.689 \\
\hline $\begin{array}{l}\text { 5.core } 9.1 \% \mathrm{GeO}_{2}, 7.7 \% \\
\mathrm{~B}_{2} \mathrm{O}_{3}, 83.2 \% \mathrm{Si} \mathrm{O}_{2} \\
\text { shell } 2.2 \% \mathrm{GeO}_{2}, 3.3 \% \mathrm{~B}_{2} \mathrm{O}_{3} \text {, } \\
94.5 \% \mathrm{SiO}_{2}\end{array}$ & 3.782487 & 0.177092 & 0.553 & 0.034728 & 3.272 \\
\hline $\begin{array}{l}\text { 6.core } 4.03 \% \mathrm{GeO}_{2}, 9.7 \% \\
\mathrm{~B}_{2} \mathrm{O}_{3}, 86.27 \% \mathrm{SiO}_{2} \\
\text { shell } 1 \% \mathrm{~F}, 99 \% \mathrm{SiO}_{2}\end{array}$ & 3.652326 & 0.170998 & 0.553 & 0.034728 & 3.371 \\
\hline $\begin{array}{l}\text { 7.core } 0.1 \% \mathrm{GeO}_{2}, 5.4 \% \\
\mathrm{~B}_{2} \mathrm{O}_{3}, 94.5 \% \mathrm{Si} \mathrm{O}_{2} \\
\text { shell } 1 \% \mathrm{~F}, 99 \% \mathrm{SiO}_{2}\end{array}$ & 2.981806 & 0.139605 & 0.553 & 0.034728 & 4.12 \\
\hline
\end{tabular}

A special case of (SSMOF) is optic fiber with ordered rotated of glass microstructure (ORGM), in this case (A $\rightarrow \infty)$, thus there is no power exchange between waves ( $H E_{11}^{e}$ and $H E_{11}^{o}$ ) [6]. Expressions for the shape and size calculation of the ordinary $\left(S^{o}\right)$ and extraordinary $\left(S^{e}\right)$ waves in the cylindrical coordinate system are obtained:

$S^{o}=S_{o}\left(1+\frac{\pi \cdot r_{1}}{p} \sin \varphi\right)$

$S^{e}=S_{o}\left(1+\frac{\pi \cdot r_{1}}{p} \cos \varphi\right)$

Where $S_{o}$-is the cross section of a round beam of rays with radius $r_{1}$ in an isotropic optical fiber.

The performed calculations for $\left(S^{o}, S^{e}, S_{o}\right)$ with twist step $\rho=\pi . r_{1}$ on figure. 4 indicate the beam expansion in the direction of dielectric constant increasing.
We can see the shift of the extraordinary ray towards the coordinate $\varphi=90^{\circ}$ and the ordinary ray towards the coordinate $\varphi=0^{\circ}$. The obtained ray divergence can be used for spatial dilution of $\left(H E_{11}^{e}\right.$ and $\left.H E_{11}^{o}\right)$ waves at the output of an anisotropic optical fiber with (ORGM) 


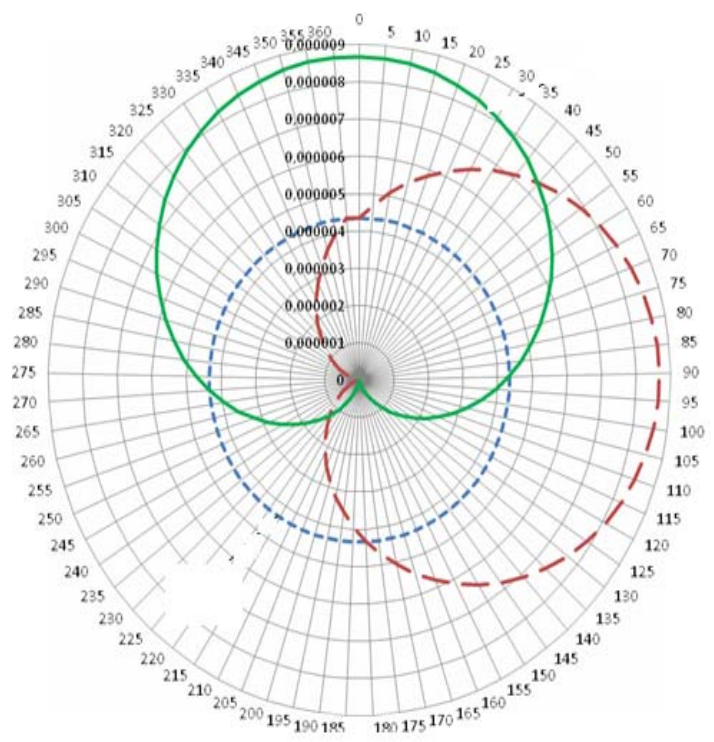

Fig.4: The ray cross-sectional shapes

Where the blue color represent $S_{o}\left(H E_{11}\right)$, and the brown color represent $S^{e}\left(H E_{11}^{e}\right)$ in isotropic optical fiber, the green color represent $S^{o}\left(H E_{11}^{o}\right)$ in anisotropic optical fiber.

From simplicity point of view in realizing the uniform power separation based on an anisotropic (SSMOF), the cases $\mathrm{A}=0$ (bent along the arc of the circle of fiber waveguide) and $A>2$ are the optimal, since ordinary and extraordinary waves propagate autonomously without exchange of powers, and therefore, there are no strict requirements for determining the length of the (SSMOF) section, where it is necessary to dilute both beams. And if the condition $\beta_{o}=\beta_{e}=45^{\circ}$ is satisfied, according to (1), the power of the $H E_{11}^{o}$ wave is equal to the power of the $H E_{11}^{e}$ wave.

\section{IV.CONCLUSION}

We can conclude that results and analysis of this paper indicate the possibility of implementing directional couplers, frequency-selective filters based on the anisotropy properties due to photo elasticity of optical fiber, it was determined the method for calculating the parameters of the (SSMOF) for using in optical fiber system. And the possibility to calculate the propagation Constants and the waveguide dispersion of the fundamental modes in an anisotropic dielectric waveguide and the distributions of the fields of these modes. The obtained data can be used also as a reference material for the construction of splitters based on angular dispersion.

\section{REFERENCES}

[1] AL-Gawagzeh, M. Y, 2016. Possibility of Minimizing the Effect of Transfer Parameters on the Transmitted Signal in the Spiral Optical Fiber. Int. J. of Communications, 1: pp.205-209.

[2] Makarov,T. V, 2004. Nonreciprocal effect waves and signals in single mode bended on spiral optical fiber.UNEERT, 1: pp 2329.

[3] Makarov,T. V, 2005.The transfer characteristic of bending optical fiber. UNEERT, 2: pp29-34.

[4] Arnold,J. M, 2001.Anisotropic Effect in non-linear optical waveguides. J.of wave motion, 34: pp.339-352.

[5] Zhock, N. P, 2010.Effective permittivity of a statistically inhomogeneous medium with strong permittivity fluctuations. Progress in Electromagnetic Research, 44: pp.169-195.

[6] AL-Gawagzeh, M. Y, 2009.Research the Dielectric permittivity of optical fiber. J.of electronic and electrical engineering, 89:pp. 45-49.

[7] AL-Gawagzeh, M. Y, AL-Hadidi, M. R, Razooq, R.S, 2009.Polarized modes Dispersion in Anisotropic Optical Fiber Communication lines. Wseas Transaction on Communication, 8: pp.11-20.

[8] AL-Gawagzeh, M. Y, AL-Hadidi, M. R, 2014.Chromatic dispersion in fiber optic with ordered rotating of glass microstructure (ORGM). J.of Optoelectronics and advanced materials-Rapid Communications, 8:pp.67-71.

[9] AL-Gawagzeh, M. Y, 2018.Studying the Polarization Dispersion in the Bended on Spiral Optical Waveguide. Journal of Engineering and Applied Sciences, Volume 13,issue 18: pp. 4971-4975.

[10] Iorgach, O. V, 2011. Losses on the Non-uniform Optical Fiber Cables. Journal of Foto- Electron, 11:pp. 61-64.

[11] Peter, C. E, 2008.Effect of Production Process on the Optical Fiber Properties. Electrical communication Journal, 2: pp.27-32.

[12] Kirill Zeyde, Vadim Sharov, Mikhail Ronkin, Guided Microwaves Electromagnetic Drag over the Sensitivity Threshold Experimental Observation WSEAS Transactions on Communications, Volume 18, 2019, pp. 191-202 